\title{
Quantifications of In Vivo Labeled Stem Cells Based on Measurements of Magnetic Moments
}

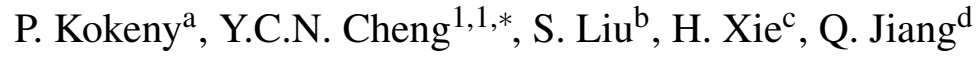 \\ ${ }^{a}$ Department of Radiology, Wayne State University, Detroit, MI 48201 \\ ${ }^{b}$ The MRI Institute for Biomedical Research, 761 Lucerne Avenue, Waterloo, ON, Canada \\ ${ }^{c}$ Department of Physics, Wayne State University, Detroit, MI 48201 \\ ${ }^{d}$ Department of Neurology, Henry Ford Health System, Detroit, MI 48202
}

\begin{abstract}
Cells labeled by super paramagnetic iron-oxide (SPIO) nanoparticles are more easily seen in gradient echo MR images, but it has not been shown that the amount of nanoparticles or the number of cells can be directly quantified from MR images. This work utilizes a previously developed and improved Complex Image Summation around a Spherical or Cylindrical Object (CISSCO) method to quantify the magnetic moments of several clusters of SPIO nanoparticle labeled cells from archived rat brain images. With the knowledge of mass magnetization of the cell labeling agent and cell iron uptake, the number of cells in each nanoparticle cluster can be determined. Using a high pass filter with a reasonable size has little effect on each measured magnetic moment from the CISSCO method. These procedures and quantitative results may help improve the efficacy of cell-based treatments in vivo.
\end{abstract}

Keywords: suscpetibility, MRI, stem cells, magnetic moment, quantification

${ }^{*}$ Corresponding author
Email addresses: kokenymri@gmail.com (P. Kokeny), yxc16@wayne.edu (Y.C.N. Cheng)

Preprint submitted to Nuclear Physics B

August 4, 2016

(C) 2016. This manuscript version is made available under the Elsevier user license

http://www.elsevier.com/open-access/userlicense/1.0/ 


\section{Introduction}

Cell-based treatments have shown promising results in neurological diseases in laboratory animals [1, 2] and patients [3, 4]. Therapeutic benefit using neural progenitor cells (NPCs) depends on the migration and localizations of the grafted cells within the target tissue [5, 6, 7]. Magnetic resonance imaging (MRI) has demonstrated its ability in monitoring cell migration and distributions using dextran-coated superparamagnetic iron oxide (SPIO) nanoparticles with transfer agents $[8,9,10,11,12,13,14,15,16,17,18,19]$. Quantification of SPIO tagged cells may open the door to more detailed studies on their behaviors after SPIO are administered into the body, yet quantification of in vivo labeled cells remains a challenge. Assessment of iron (e.g., brain iron) has typically involved the measurement of proton transverse relaxation rate, $R_{2}$ [20, 21, 22, 23, 24, 25]. Several studies have observed a relationship between $R_{2}$ (or $R_{2}^{*}$ ) and labeled cell concentration in vitro [26, 27] . However, the relationship between $R_{2}$ and labeled cell concentration is much more complicated in vivo and may involve diffusion [28]. Quantifications of labeled cell concentrations using $R_{2}$ and diffusion require the removal of background effects induced by neurological diseases [28].

Previously, Del Gratta et al. [29] had estimated numbers of cells ex vivo using a Superconducting Quantum Interference Device (SQUID) magnetometer. In this work, we propose to use the Complex Image Summation around a Spherical or Cylindrical Object (CISSCO) method [30] to quantify the magnetic moment of SPIO nanoparticle tagged stem cells in a local cluster from MR images. In turn, we estimate the number of cells from each cluster. For the magnetic moment quantification of small objects, the CISSCO method has been tested and shown to provide good accuracy [30].

Magnetostatic theory governs that small magnetized objects, regardless of geometry, can be modeled as perfect spheres. The magnetic moment of an object can be expressed as the product of its mass magnetization and mass. Thus, given the known mass magnetization of iron nanoparticles and the quantified magnetic moment of the nanoparticle cluster, the mass of nanoparticles in a local cluster can be calculated. Further, if the cellular iron uptake is known, the mass of nanoparticles can be used to derive the number of cells. In order to apply the CISSCO method to in vivo images, we first simulate and study the effect of high-pass (HP) filters and systematic errors on magnetic moment quantifications. High-pass filtering [31] has been a relatively easy and effective method for eliminating background fields from phase images. Next, an improved version of the CISSCO method for in vivo applications is tested on simulations and then applied to six clusters of cells in several existing rat brain images.

\section{Materials and Methods}

\subsection{Simulations}

We simulated several spheres that represent cell clusters, which in reality contains numerous nanoparticles. Given various magnetic moments and radii of simulated spheres, each simulation was forward modeled on a $1024^{3}$ matrix and cropped down to $32^{3}$ in the spatial frequency domain. Detailed procedures were given in [30]. For display purposes and analyses of HP filters, each of the 32 slices was zero-filled (zero phase value and a predefined constant magnitude intensity outside the $32 \times 32$ matrix) in image space to $256^{2}$. A total of 15 spheres were simulated with targeted effective magnetic moments $(p)$ of $8,15,20,30$, and 100 radian pixel $^{3}$ and radii $(a)$ of 1 , 
2 , and 3 pixels in the $32^{3}$ matrix. The effective magnetic moment of each simulated sphere from CISSCO has been defined as $p=0.5 \chi \Delta \chi B_{0} T_{E} V$, where $\gamma=42.58 \mathrm{MHz} / \mathrm{T}, B_{0}$ is the main field, $T_{E}$ is the echo time, $\Delta \chi$ is the susceptibility difference between the sphere and its surrounding, and $V$ is the volume of the sphere. As $0.5 \chi \Delta \chi B_{0} T_{E}$ is a product of 4 parameters and can be rewritten as $p$ divided by $a^{3}$, it is more general to consider the radius and the effective magnetic moment for each simulation (rather than each individual parameter). This effective magnetic moment has a unit of radian-pixel ${ }^{3}$ if the volume of the sphere is measured in terms of pixel ${ }^{3}$ directly from images. In the case of nanoparticles in tissues, $\Delta \chi B_{0} V$ can be approximated by $\mu_{0} \mu$ [32], where $\mu_{0}=4 \pi \times 10^{-7} \mathrm{~Wb} /(\mathrm{A} \cdot \mathrm{m})$ and $\mu$ is the overall magnetic moment of nanoparticles within a cluster in a tissue and has a unit of $\mathrm{A} \cdot \mathrm{m}^{2}$. For simplicity, $p$ refers to the "magnetic moment" hereafter. No Gaussian noise was added to any of the simulations in order to examine the effect of the HP filter and its systematic errors. All magnetic moment quantifications from CISSCO were performed using codes developed in Visual $\mathrm{C}++$.

\subsection{CISSCO Procedure}

The procedure of magnetic moment quantifications using CISSCO has been given in [30]. Briefly, each voxel is first interpolated into 1000 subvoxels for subsequent steps. Second, the center of an object of interest is identified by minimizing the real part of the overall signal summed within an arbitrary sphere chosen by the user. Third, the magnetic moment $p$ of the object is solved from the following equation

$$
\operatorname{Re}\left(S_{1}-S_{2}\right) \operatorname{Re}\left(f_{23}(p)\right)=\operatorname{Re}\left(S_{2}-S_{3}\right) \operatorname{Re}\left(f_{12}(p)\right)
$$

where $S_{1}, S_{2}$, and $S_{3}$ are the complex sums within three concentric pseudo spheres with radii $R_{1}$, $R_{2}$, and $R_{3}$ defined by the user, and $f_{12}(p)$ and $f_{23}(p)$ are analytical functions given by equations 6,9 , and 10 of [30] and shown below. They represent the normalized theoretical sums of complex signals within pseudo shells defined by the three radii. Note that $R_{1}>R_{2}>R_{3}$.

$$
f_{i j}(p) \equiv R_{i}^{3} \int_{1}^{\frac{R_{i}^{3}}{R_{j}^{3}}} \frac{d x}{x^{2}}\left(2 e^{i x \frac{p}{R_{i}^{3}}}+e^{-2 i x \frac{p}{R_{i}^{3}}}\right)+\int_{-1}^{2} \frac{d x}{x^{2}}[2-(2-x) \sqrt{1+x}]\left(R_{i}^{3} e^{-i x \frac{p}{R_{i}^{3}}}-R_{j}^{3} e^{-i x \frac{p}{R_{j}^{3}}}\right)
$$

\subsection{Improved CISSCO Approach}

As an in vivo object can be subject to local background magnetic fields induced from other nearby tissues, it is important to correct this problem in the CISSCO method. Given the fact that we are interested in small objects, we may approximate those local background fields as a constant local field around each object. This local constant background field may affect the determination of the object center in CISSCO as well as the quantification of the magnetic moment. To study the effect analytically, we have added a constant background phase $\phi_{b k g}$ to signal $S$ defined in [30]. The signal becomes $S \times \exp \left(i \phi_{b k g}\right)$. Regardless of the value of $\phi_{b k g}$, it should be clear from [30] that first derivatives and cross terms of second derivatives of $S \times \exp \left(i \phi_{b k g}\right)$ are all zero, when the center of the object is identified at $r_{0}=0$. The remaining second derivatives can be written down according to Eq. 27 of [30]. 


$$
\begin{aligned}
\left.\frac{\partial^{2} S e^{i \phi_{b k g}}}{\partial x_{0}^{2}}\right|_{r_{0}=0}= & 2 \pi \rho_{0} a e^{i \phi_{b k g}}\left[\int_{0}^{1} d x\left(3 x^{2}-1\right) f_{a}-3 \phi_{a}^{1 / 3} \int_{\phi_{R}}^{\phi_{a}} \phi^{2 / 3} d \phi \int_{0}^{1} d x\left(5 x^{2}-1\right)^{2}\left(1-x^{2}\right) f_{\phi}\right. \\
& \left.+3 i \phi_{a} \int_{0}^{1} d x\left(7 x^{2}-1\right)\left(1-x^{2}\right) f_{a}+i \phi_{a}^{1 / 3} \int_{\phi_{R}}^{\phi_{a}} \frac{d \phi}{\phi^{1 / 3}} \int_{0}^{1} d x\left(35 x^{4}-30 x^{2}+3\right) f_{\phi}\right] \\
\left.\frac{\partial^{2} S e^{i \phi_{b k g}}}{\partial y_{0}^{2}}\right|_{r_{0}=0}= & \left.\frac{\partial^{2} S e^{i \phi_{b k g}}}{\partial x_{0}^{2}}\right|_{r_{0}=0} \\
\left.\frac{\partial^{2} S e^{i \phi_{b k g}}}{\partial z_{0}^{2}}\right|_{r_{0}=0}= & -4 \pi \rho_{0} a e^{i \phi_{b k g}}\left[\int_{0}^{1} d x\left(3 x^{2}-1\right) f_{a}+3 \phi_{a}^{1 / 3} \int_{\phi_{R}}^{\phi_{a}} \phi^{2 / 3} d \phi \int_{0}^{1} d x\left(3-5 x^{2}\right)^{2} x^{2} f_{\phi}\right. \\
& \left.+3 i \phi_{a} \int_{0}^{1} d x\left(5-7 x^{2}\right) x^{2} f_{a}+i \phi_{a}^{1 / 3} \int_{\phi_{R}}^{\phi_{a}} \frac{d \phi}{\phi^{1 / 3}} \int_{0}^{1} d x\left(35 x^{4}-30 x^{2}+3\right) f_{\phi}\right]
\end{aligned}
$$

where $\rho_{0}$ is the spin density of the tissue around the object, $f_{a} \equiv \exp \left\{-i \phi_{a}\left(3 x^{2}-1\right)\right\}, f_{\phi} \equiv$ $\exp \left\{-i \phi\left(3 x^{2}-1\right)\right\}, \phi_{a} \equiv p / a^{3}, \phi_{R} \equiv p / R^{3}$, and $R$ is the radius of the sphere that a user chooses to identify the center of the object. The goal is to determine, under what $\phi_{b k g}, \phi_{a}$, and $\phi_{R}$ values that real parts of all three second derivatives are positive. We consider $\phi_{b k g}$ ranging from -1.5 radians to +1 radian in an increment of 0.1 radian for a series of numerical evaluations. For each value of $\phi_{b k g}$, we have varied $\phi_{a}$ from 0.1 to $3 \pi$ radians and $\phi_{R}$ from 0.1 to $\pi$ radians in order to examine the signs of those second derivatives. As $\phi_{R}$ indirectly defines the radius of the sphere that the user chooses to find the center of the object, this gives a scale invariant variable to use, rather than to use the magnetic moment $p$ in these calculations. The range of $\phi_{R}$ under consideration is always between 0 and $\pi$ [30]. If $\phi_{a}$ is larger than $3 \pi$, given the dephasing effect around the object of interest, in order to identify its center, the object can be substituted by a larger object (but the same magnetic moment) such that its $\phi_{a}$ is less than $3 \pi$.

Next, in order to eliminate this constant field term when we solve for the magnetic moment, based on the original derivations of the CISSCO method in [30], we can consider the magnitude squared of the complex signals rather than their real parts. We thus have

$$
\left|S_{1}-S_{2}\right|^{2}\left|f_{23}(p)\right|^{2}=\left|S_{2}-S_{3}\right|^{2}\left|f_{12}(p)\right|^{2}
$$

It should be clear from Eq. 4 that the local constant phase term will not affect the quantification of the magnetic moment. To verify this, we will add a constant background phase ranging from -1 to 1 radian in increments of 0.1 radian, to a simulated sphere with a radius of 1 pixel and a magnetic moment of 20 radian $\cdot$ pixel $^{3}$. Each case will be quantified by both the original CISSCO method and the improved method. This alternate approach also requires rederiving the uncertainty of the quantified magnetic moment, including both the Gaussian noise and systematic error. This has been accomplished using a standard error propagation approach (See Appendix).

\subsection{HP Filter}

It was known that HP filters could affect phase values from large objects but it was not clear how HP filters could affect the magnetic moment quantification from CISSCO. Thus, high-pass filters with sizes of $16 \times 16,32 \times 32$, and $64 \times 64$, and with an additional Hanning filter multiplied to each HP filter in k-space were applied to each slice of the simulated data. The magnetic moment 


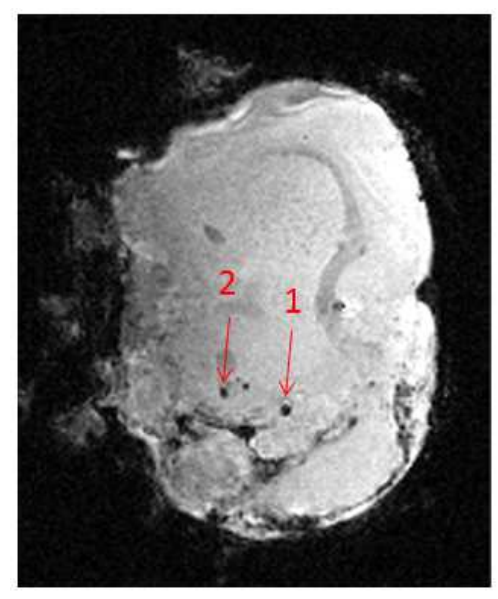

(a)

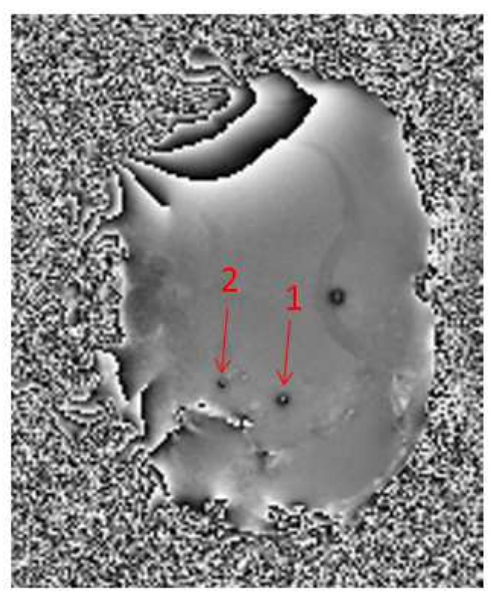

(b)

Figure 1: Example (a) magnitude and (b) phase image of nanoparticle labeled cell clusters in a rat. Clusters that were quantified are identified by red arrows.

of each simulated sphere, before and after the applications of the HP filters, was quantified using CISSCO. This was to evaluate systematic errors purely from HP filters, rather than from Gibbs ringing or the partial volume effect. The radii of the three pseudo spheres used in CISSCO were chosen at least 0.5 pixel away from the surface of each spherical object and at least one pixel apart between any two pseudo spheres [30].

\subsection{In Vivo Data and Analyses}

We applied a $32 \times 32 \mathrm{HP}$ filter to four sets of archived 3D gradient echo rat images acquired in 2008 based on a stroke model performed in [28]. Neural progenitor cells were labeled using a Ferumoxide-Sulfate solution and were injected into those rat brains. Six isolated nanoparticle clusters from the four datasets were quantified with the CISSCO method. Two example clusters are shown in Fig. 1 . The imaging parameters were $T_{E}=10 \mathrm{~ms}, T_{R}=30 \mathrm{~ms}, B_{0}=7 \mathrm{~T}$, in-plane field of view $=32 \mathrm{~mm} \times 32 \mathrm{~mm}$, image resolution $=0.0625 \mathrm{~mm} \times 0.125 \mathrm{~mm} \times 0.25 \mathrm{~mm}$, matrix size $=$ $512 \times 256 \times 64$, slab thickness $=16 \mathrm{~mm}$, flip angle $=25^{\circ}$, number of averages $=4$, and scan time $=32 \mathrm{mins} 46 \mathrm{sec}$. Original $\mathrm{k}$-space data were used for image reconstructions. The reconstructed images were further interpolated to $0.125 \mathrm{~mm}$ isotropic resolution for CISSCO analyses. A $T_{2}$ weighted spin echo dataset with the same in-plane resolution was also obtained with the intent to estimate the volume of each cluster. However, clusters in spin echo images were unidentifiable. As the slice thickness of the spin echo images $(0.8 \mathrm{~mm})$ was about three times that of the gradient echo images, and spin echo images had sufficient signal-to-noise ratios in images, these facts implied that the overall volume of nanoparticles in each cluster was still much smaller than a voxel in gradient echo images.

The number of cells in each cluster was calculated from the following equation, with the result rounded to the nearest integer.

Number of cells $=\mu\left(\mathrm{A} \cdot \mathrm{m}^{2}\right) \times \frac{1}{\text { Ferumoxide mass magnetization }}\left(\frac{\mathrm{kg}}{\mathrm{A} \cdot \mathrm{m}^{2}}\right) \times \frac{1}{\text { cell iron uptake }}\left(\frac{\text { cell }}{\mathrm{kg} \mathrm{Fe}}\right)$ 
where $\mu$ was calculated from the measured magnetic moment $p=0.5 \not T_{E} \mu_{0} \mu$. The saturation mass magnetization of Ferumoxide is $93.6 \pm 1.6 \mathrm{~A} \cdot \mathrm{m}^{2} / \mathrm{kg}$ iron [33]. Values for cell iron uptake depend on the cell labeling agent and process, along with the type of cell being used. We chose $14.5 \mathrm{pg} / \mathrm{cell}$ from Panizzo et al. [34], as they used the same labeling agent, process, and cell type provided in [28].

\section{Results}

\subsection{HP Filter}

Simulations showed that, as the size of the applied HP filter increases, the quantified magnetic moment decreases (Fig. 2). The error is larger when either the magnetic moment or the radius of the simulated sphere is larger. The percentage differences in magnetic moment quantifications caused by the $32 \times 32$ HP filter can vary by a large range depending on the magnetic moment and radius of the sphere (Fig. 2d). For spheres with radii of 2 or 3 pixels, we observed a minimal value (i.e., error) in Fig. 2 d, at the magnetic moment of about 30 radian $\cdot$ pixel $^{3}$. When the magnetic moment is smaller, the error increases. This is due to less than $\pi$ radians of phase values outside the object such that other systematic errors affect the results more.

\subsection{Improved CISSCO Approach}

Figure 3 indicates the largest radius $R$ within which complex signals can be summed over such that the center of an object of interest can be identified through Eq. 3. With a given local constant background phase, the radius $R$ of the pseudo sphere needs to be chosen sufficiently large such that $\left|p / R^{3}\right|$ is less than the maximal $\left|\phi_{R}\right|$ value shown in Fig. 3.

Figure 4 shows results for quantification of a simulated sphere with a radius of 1 pixel and a true magnetic moment of 20 radian pixel $^{3}$ over a range of background phases, using both the original and improved CISSCO method. When the original method was used, the quantified magnetic moment decreased from 24.3 radian $\cdot \mathrm{pixel}^{3}$ to $14.7 \mathrm{radian} \cdot \mathrm{pixel}^{3}$ as the background phase increased. On the other hand, results from the improved method remained at 19.95 radian.pixel ${ }^{3}$ for each background phase. It is clear that the improved approach is not at all susceptible to the added local constant background phase.

\subsection{Number of Cells from In Vivo Data}

Table 1 summarizes results from six stem cell clusters quantified from the CISSCO method. Each result was adjusted based on findings from the simulated HP filter results. As the uncertainty of mass magnetization of Ferumoxide is about $1.7 \%$ but the uncertainty of cell iron uptake is not known, the percentage error of each cell count is the sum of $1.7 \%$ and the percentage error estimated from CISSCO (third column in Table 1). All but one quantified results had less than $10 \%$ uncertainty. Table 1 also indicates that if one cell contains $14.5 \mathrm{pg}$ of iron, then the quantified magnetic moment would be roughly 0.19 radian $\cdot \operatorname{pixel}^{3}$. Thus detecting one cell would require a higher image resolution than the current resolution. On the other hand, Heyn et al. [35] had a cellular iron uptake of $43.3 \mathrm{pg} / \mathrm{cell}$ and they were able to visually detect one cell. This $43.3 \mathrm{pg}$ of iron per cell translates to a magnetic moment of roughly 0.56 radian $\cdot$ pixel $^{3}$, which is about the lower limit that our CISSCO method can quantify with an uncertainty of roughly $100 \%$. In general, other studies $[36,35,37,38]$ also have cellular iron uptakes at the same order of magnitude. Thus, their images can also be analyzed with our method. 


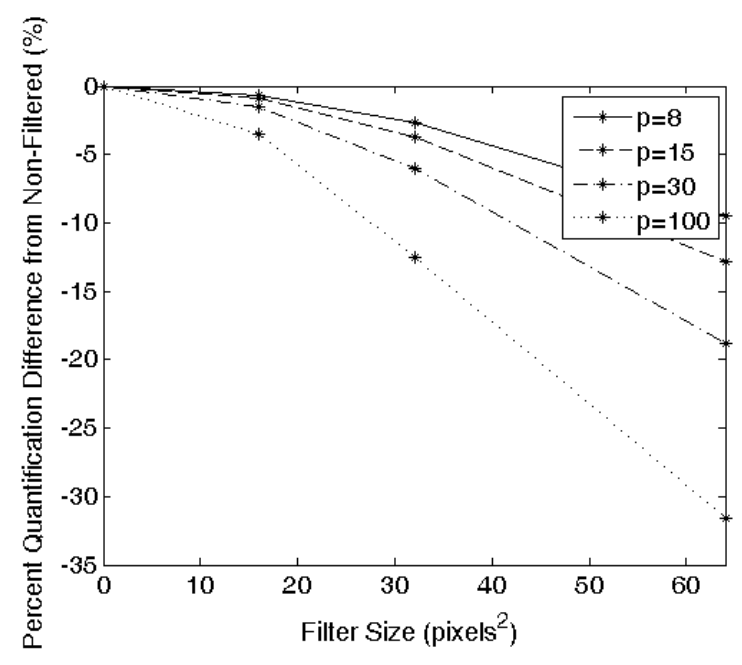

(a)

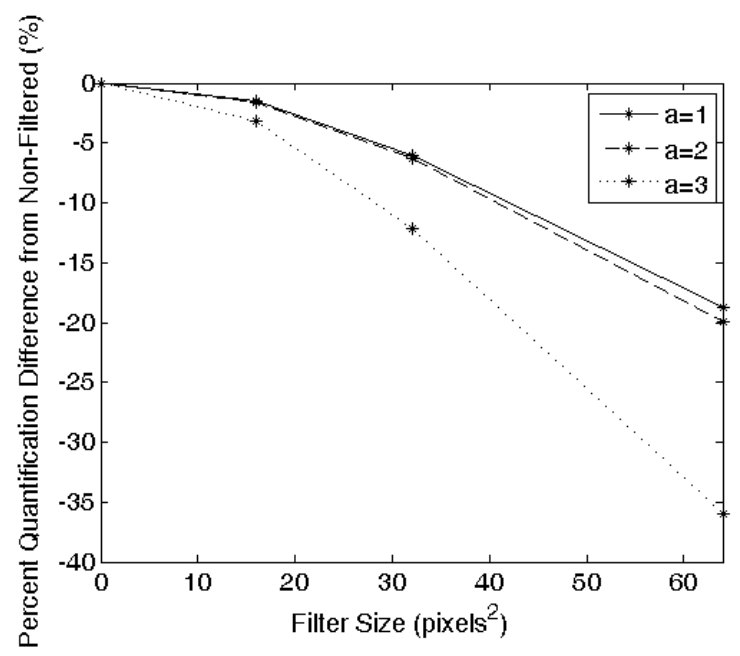

(c)

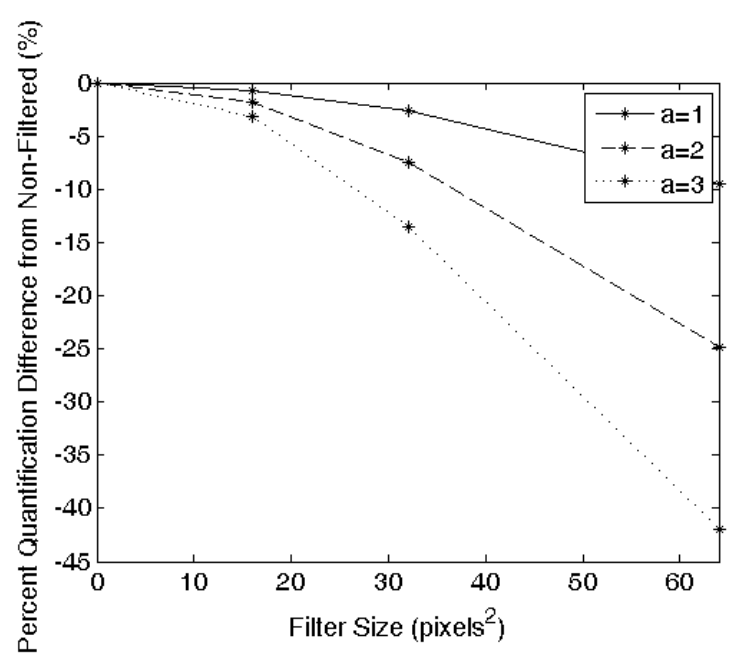

(b)

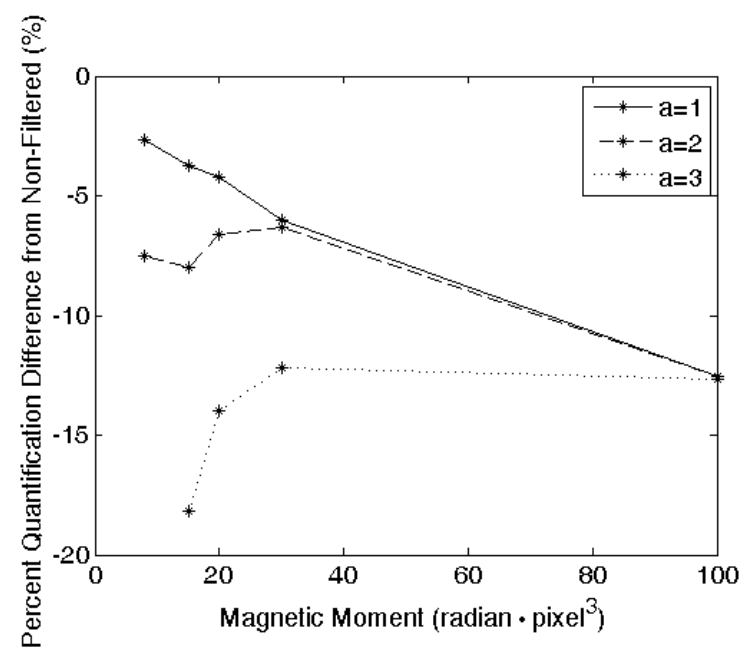

(d)

Figure 2: Percentage differences in magnetic moment quantifications as a function of filter sizes for simulated spheres with (a) a radius of 1 pixel, (b) a fixed magnetic moment of 8 radian $\cdot$ pixel $^{3}$, and (c) a fixed magnetic moment of 30 radian pixel $^{3}$. (d) Percentage differences in magnetic moment quantifications as a function of magnetic moments. These results are from a fixed $32 \times 32 \mathrm{HP}$ filter but with different sphere sizes. Lines connecting data points do not have specific meanings. 


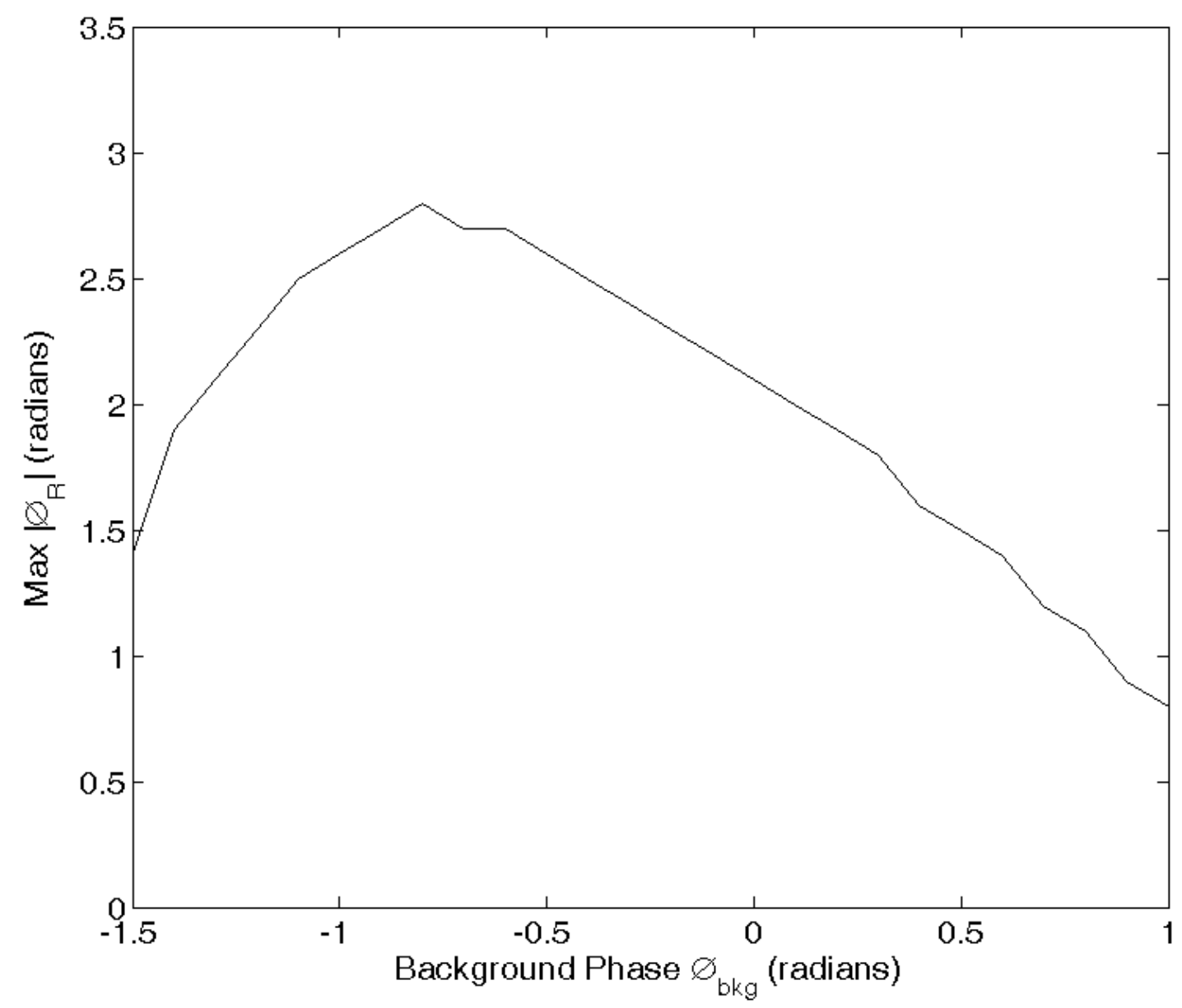

Figure 3: Maximal $\left|\phi_{R}\right|$ value as a function of the local constant background phase, $\phi_{b k g}$. As $\phi_{R}$ indicates the phase values induced from the object on the equatorial plane, maximal $\left|\phi_{R}\right|$ associated with a given $\phi_{b k g}$ value implies how large the radius $R$ should be chosen for the determination of the object center.

\begin{tabular}{|c|c|c|c|c|c|r|}
\hline Cluster & $p_{H P}^{\prime}$ & $\delta p^{\prime} / p^{\prime}(\%)$ & $p_{\text {adj }}^{\prime}$ & Cells & $\eta_{12}(\%)$ & $\eta_{23}(\%)$ \\
\hline 1 & 12.2 & 3.6 & 13.0 & 70 & -0.5 & 0.3 \\
\hline 2 & 5.38 & 2.4 & 5.72 & 31 & -1.1 & 3.4 \\
\hline 3 & 11.5 & 3.1 & 12.2 & 66 & -0.5 & -5.2 \\
\hline 4 & 28.8 & 1.2 & 30.6 & 164 & -1.4 & 2.4 \\
\hline 5 & 7.66 & 8.8 & 8.15 & 44 & -0.3 & 7.4 \\
\hline 6 & 21.7 & 0.8 & 23.1 & 124 & -1.6 & 0.9 \\
\hline
\end{tabular}

Table 1: Quantified results of six nanoparticle clusters. The second column $p_{H P}^{\prime}$ represents each measured magnetic moment from CISSCO after images have been high-pass filtered. The unit of each measurement is radian-pixel ${ }^{3}$. The third column lists the uncertainty (in percentage) of each quantified magnetic moment. The fourth column lists the HP filter corrected magnetic moment based on the value of $p_{H P}^{\prime}$ and the curve of $a=1$ in Fig. $2 \mathrm{~d}$. The fifth column lists the number of cells calculated from the fourth column. The last two columns give the systematic errors from the magnitude squared procedure for each concentric shell used in the CISSCO method. Partial volume effects due to non-isotropic image resolutions are not included in these systematic errors. 


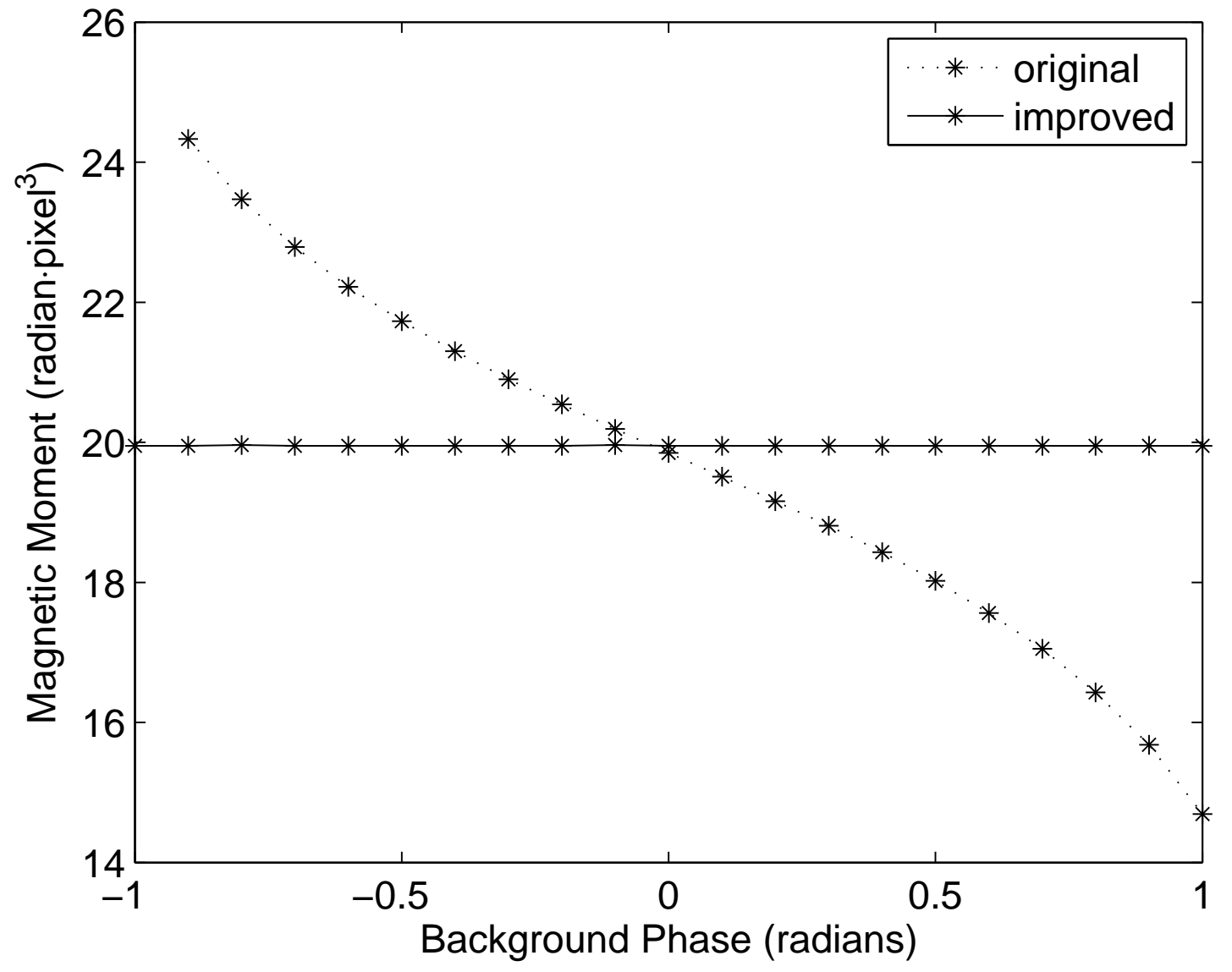

Figure 4: Quantified magnetic moments as a function of the local constant background phase using both the original (dotted line) and improved (solid line) CISSCO method. The improved method is completely unaffected by the addition of the background phase. 


\section{Discussion}

Our work has demonstrated that magnetic moments of isolated clusters of nanoparticle labeled cells can be accurately quantified from the applications of the high pass filter and the improved CISSCO method. While our results were not validated by any histological method, several issues should be realized with performing such a validation. Although cell concentrations measured by MRI and histology were highly correlated, a histological approach has its own uncertainties. Each quantified number of labeled cells can vary by a factor of 4 , indicated from private records in the work of [28]. Those uncertainties depend on cell size, cell division, labeled macrophage, cell death, image resolution, and also are based on the assumption that cells are uniformly distributed throughout the cluster. As a result, there is a strong need of a better method for the quantification of number of labeled cells, especially in vivo.

Although a larger magnetic moment will have a smaller uncertainty estimated from the CISSCO method, it will nonetheless have a larger error after the application of the HP filter. In addition, the larger the HP filter size is, the worse the underestimation of the result will be. As we are not able to spot each nanoparticle cluster from spin echo images, the overall volume of nanoparticles in a cluster has to be much smaller than a voxel. This is also consistent with small volumes estimated from quantified magnetic moments (Table 1) divided by a large magnetization of nanoparticles. These facts suggest that the $32 \times 32 \mathrm{HP}$ filter has little effects on our in vivo magnetic moment measurements. If we want to make some adjustments, we can use the curve in Fig. 2d, when the radius of the object $a$ is 1 pixel.

While systematic error is ultimately unavoidable, it can be minimized by scanning with an isotropic image resolution. Although the actual geometry of each cluster is not a perfect sphere, magnetostatic theory tells us that the magnetic moment of such a small object can be well approximated by that from a sphere. In order to improve the accuracy of each measurement, it is also important to re-measure the mass magnetization of nanoparticles and cell iron uptake used in a particular study. These two quantities can be measured in ex vivo settings.

Our results show that the improved CISSCO method is better than the original method, as constant background phase has no effect on quantifications. For use in center identification, our criteria on the radius $R$ must be followed. Even though the magnetic moment $p$ is not known beforehand, $p / R^{3}$ is the theoretical phase value on the equatorial plane around the spherical object and thus is roughly known from phase images. Because of that, and if the local constant background phase $\phi_{b k g}$ can also be estimated from phase images, then we can properly choose the radius $R$ based on Fig. 3 for the determination of the object center. From a different consideration, if $\left|\phi_{b k g}\right|$ is relatively large, say more than 0.3 or 0.5 radian, we can consider subtracting a constant phase value around the object such that the remanent local background phase is within \pm 0.3 or \pm 0.5 radian. This will allow us to reduce the radius $R$, keep the pseudo sphere compact, and effectively identify the center of the object.

\section{Conclusions}

We have presented an improved method of magnetic moment quantification of labeled stem cell clusters from archived in vivo images. For this purpose, our simulations confirm that images filtered by a $32 \times 32$ high-pass filter only lead to slightly underestimated results. This improved CISSCO method is also insensitive to the existence of local background field. Although we have 
not directly validated our method with histological results, past records indicate that histological results have large uncertainties. A better method of measuring number of labeled cells is needed. On the other hand, from the quantified magnetic moment, we can calculate the number of cells in each cluster to a reasonable accuracy. This offers a new potential for the measurement of the number of cells in vivo.

\section{Acknowledgment}

This work was supported in part by the Department of Radiology at Wayne State University as well as grants NIH/NHLBI 108230-01A2, NIH/NINDS 64134, and DOD/USAMRAA W81XWH12-1-0522. The content is solely the responsibility of the authors and does not necessarily represent the official views of the Department of Defense.

[1] Z. G. Zhang and M. Chopp, "Neurorestorative therapies for stroke: underlying mechanisms and translation to the clinic," Lancet Neurol, vol. 8, no. 5, pp. 491-500, 2009.

[2] Y. Li and M. Chopp, "Marrow stromal cell transplantation in stroke and traumatic brain injury," Neurosci Lett, vol. 456, no. 3, pp. 120-3, 2009.

[3] H. R. Aghayan, B. Arjmand, M. Yaghoubi, M. Moradi-Lakeh, H. Kashani, and F. Shokraneh, "Clinical outcome of autologous mononuclear cells transplantation for spinal cord injury: a systematic review and meta-analysis," Med J Islam Repub Iran, vol. 28, p. 112, 2014.

[4] T. R. Doeppner and D. M. Hermann, "Stem cell-based treatments against stroke: observations from human proof-of-concept studies and considerations regarding clinical applicability," Front Cell Neurosci, vol. 8, p. 357, 2014.

[5] S. B. Dunnett, A. Bjorklund, and O. Lindvall, "Cell therapy in parkinson's disease - stop or go?," Nat Rev Neurosci, vol. 2, no. 5, pp. 365-9, 2001.

[6] Q. Jiang, Z. G. Zhang, G. L. Ding, B. Silver, L. Zhang, H. Meng, M. Lu, D. S. PourabdillahNejed, L. Wang, S. Savant-Bhonsale, L. Li, H. Bagher-Ebadian, J. Hu, A. S. Arbab, P. Vanguri, J. R. Ewing, K. A. Ledbetter, and M. Chopp, "Mri detects white matter reorganization after neural progenitor cell treatment of stroke," Neuroimage, vol. 32, no. 3, pp. 1080-9, 2006.

[7] Q. Jiang, Z. G. Zhang, G. L. Ding, L. Zhang, J. R. Ewing, L. Wang, R. Zhang, L. Li, M. Lu, H. Meng, A. S. Arbab, J. Hu, Q. J. Li, D. S. Pourabdollah Nejad, H. Athiraman, and M. Chopp, "Investigation of neural progenitor cell induced angiogenesis after embolic stroke in rat using mri," Neuroimage, vol. 28, no. 3, pp. 698-707, 2005.

[8] M. Hoehn, E. Kustermann, J. Blunk, D. Wiedermann, T. Trapp, S. Wecker, M. Focking, H. Arnold, J. Hescheler, B. K. Fleischmann, W. Schwindt, and C. Buhrle, "Monitoring of implanted stem cell migration in vivo: a highly resolved in vivo magnetic resonance imaging investigation of experimental stroke in rat," Proc Natl Acad Sci U S A, vol. 99, no. 25, pp. 16267-72, 2002. 
[9] J. W. Bulte, I. D. Duncan, and J. A. Frank, "In vivo magnetic resonance tracking of magnetically labeled cells after transplantation," J Cereb Blood Flow Metab, vol. 22, no. 8, pp. 899907, 2002.

[10] J. A. Frank, B. R. Miller, A. S. Arbab, H. A. Zywicke, E. K. Jordan, B. K. Lewis, J. Bryant, L. H., and J. W. Bulte, "Clinically applicable labeling of mammalian and stem cells by combining superparamagnetic iron oxides and transfection agents," Radiology, vol. 228, no. 2, pp. 480-7, 2003.

[11] J. W. Bulte and D. L. Kraitchman, "Iron oxide mr contrast agents for molecular and cellular imaging," NMR Biomed, vol. 17, no. 7, pp. 484-99, 2004.

[12] J. W. Bulte, T. Ben-Hur, B. R. Miller, R. Mizrachi-Kol, O. Einstein, E. Reinhartz, H. A. Zywicke, T. Douglas, and J. A. Frank, "Mr microscopy of magnetically labeled neurospheres transplanted into the lewis eae rat brain," Magn Reson Med, vol. 50, no. 1, pp. 201-5, 2003.

[13] J. W. Bulte and M. De Cuyper, "Magnetoliposomes as contrast agents," Methods Enzymol, vol. 373, pp. 175-98, 2003.

[14] J. W. Bulte, T. Douglas, B. Witwer, S. C. Zhang, E. Strable, B. K. Lewis, H. Zywicke, B. Miller, P. van Gelderen, B. M. Moskowitz, I. D. Duncan, and J. A. Frank, "Magnetodendrimers allow endosomal magnetic labeling and in vivo tracking of stem cells," Nat Biotechnol, vol. 19, no. 12, pp. 1141-7, 2001.

[15] J. A. Frank, S. A. Anderson, H. Kalsih, E. K. Jordan, B. K. Lewis, G. T. Yocum, and A. S. Arbab, "Methods for magnetically labeling stem and other cells for detection by in vivo magnetic resonance imaging," Cytotherapy, vol. 6, no. 6, pp. 621-5, 2004.

[16] A. S. Arbab, G. T. Yocum, H. Kalish, E. K. Jordan, S. A. Anderson, A. Y. Khakoo, E. J. Read, and J. A. Frank, "Efficient magnetic cell labeling with protamine sulfate complexed to ferumoxides for cellular mri," Blood, vol. 104, no. 4, pp. 1217-23, 2004.

[17] A. S. Arbab, G. T. Yocum, A. M. Rad, A. Y. Khakoo, V. Fellowes, E. J. Read, and J. A. Frank, "Labeling of cells with ferumoxides-protamine sulfate complexes does not inhibit function or differentiation capacity of hematopoietic or mesenchymal stem cells," NMR Biomed, vol. 18, no. 8, pp. 553-9, 2005.

[18] M. Janowski, P. Walczak, T. Kropiwnicki, E. Jurkiewicz, K. Domanska-Janik, J. W. Bulte, B. Lukomska, and M. Roszkowski, "Long-term mri cell tracking after intraventricular delivery in a patient with global cerebral ischemia and prospects for magnetic navigation of stem cells within the csf," PLoS One, vol. 9, no. 2, p. e97631, 2014.

[19] J. W. Bulte, "In vivo mri cell tracking: clinical studies," AJR Am J Roentgenol, vol. 193, no. 2, pp. 314-25, 2009.

[20] A. Bizzi, R. A. Brooks, A. Brunetti, J. M. Hill, J. R. Alger, R. S. Miletich, T. L. Francavilla, and G. Di Chiro, "Role of iron and ferritin in mr imaging of the brain: a study in primates at different field strengths," Radiology, vol. 177, no. 1, pp. 59-65, 1990. 
[21] J. Vymazal, R. A. Brooks, N. Patronas, M. Hajek, J. W. Bulte, and G. Di Chiro, "Magnetic resonance imaging of brain iron in health and disease," J Neurol Sci, vol. 134 Suppl, pp. 1926, 1995.

[22] J. Vymazal, R. A. Brooks, C. Baumgarner, V. Tran, D. Katz, J. W. Bulte, R. Bauminger, and G. Di Chiro, "The relation between brain iron and nmr relaxation times: an in vitro study," Magn Reson Med, vol. 35, no. 1, pp. 56-61, 1996.

[23] J. Vymazal, M. Hajek, N. Patronas, J. N. Giedd, J. W. Bulte, C. Baumgarner, V. Tran, and R. A. Brooks, "The quantitative relation between t1-weighted and t2-weighted mri of normal gray matter and iron concentration," J Magn Reson Imaging, vol. 5, no. 5, pp. 554-60, 1995.

[24] C. Schenker, D. Meier, W. Wichmann, P. Boesiger, and A. Valavanis, "Age distribution and iron dependency of the 2 relaxation time in the globus pallidus and putamen," Neuroradiology, vol. 35, no. 2, pp. 119-24, 1993.

[25] R. J. Ordidge, J. M. Gorell, J. C. Deniau, R. A. Knight, and J. A. Helpern, "Assessment of relative brain iron concentrations using t2-weighted and $\mathrm{t} 2{ }^{*}$-weighted mri at 3 tesla," Magn Reson Med, vol. 32, no. 3, pp. 335-41, 1994.

[26] M. L. Zelivyanskaya, J. A. Nelson, L. Poluektova, M. Uberti, M. Mellon, H. E. Gendelman, and M. D. Boska, "Tracking superparamagnetic iron oxide labeled monocytes in brain by high-field magnetic resonance imaging," J Neurosci Res, vol. 73, no. 3, pp. 284-95, 2003.

[27] P. Mowat, F. Franconi, C. Chapon, L. Lemaire, J. Dorat, F. Hindre, J. P. Benoit, P. Richomme, and J. J. Le Jeune, "Evaluating spio-labelled cell $\mathrm{mr}$ efficiency by three-dimensional quantitative t2* mri," NMR Biomed, vol. 20, no. 1, pp. 21-7, 2007.

[28] H. Athiraman, Q. Jiang, G. L. Ding, L. Zhang, Z. G. Zhang, L. Wang, A. S. Arbab, Q. Li, S. Panda, K. Ledbetter, A. M. Rad, and M. Chopp, "Investigation of relationships between transverse relaxation rate, diffusion coefficient, and labeled cell concentration in ischemic rat brain using mri," Magn Reson Med, vol. 61, no. 3, pp. 587-94, 2009.

[29] C. Del Gratta, S. Della Penna, P. Battista, L. Di Donato, P. Vitullo, G. L. Romani, and S. Di Luzio, "Detection and counting of specific cell populations by means of magnetic markers linked to monoclonal antibodies," Phys Med Biol, vol. 40, no. 4, pp. 671-81, 1995.

[30] Y. C. Cheng, C. Y. Hsieh, R. Tackett, P. Kokeny, R. K. Regmi, and G. Lawes, "Magnetic moment quantifications of small spherical objects in mri," Magn Reson Imaging, vol. 33, no. 6 , pp. 829-39, 2015.

[31] R. J. Ogg, J. W. Langston, E. M. Haacke, R. G. Steen, and J. S. Taylor, "The correlation between phase shifts in gradient-echo mr images and regional brain iron concentration," Magn Reson Imaging, vol. 17, no. 8, pp. 1141-8, 1999.

[32] Y. Shen, Y. C. Cheng, G. Lawes, J. Neelavalli, C. Sudakar, R. Tackett, H. P. Ramnath, and E. M. Haacke, "Quantifying magnetic nanoparticles in non-steady flow by mri," MAGMA, vol. 21, no. 5, pp. 345-56, 2008. 
[33] C. W. Jung and P. Jacobs, "Physical and chemical properties of superparamagnetic iron oxide mr contrast agents: ferumoxides, ferumoxtran, ferumoxsil," Magn Reson Imaging, vol. 13, no. 5, pp. 661-74, 1995.

[34] R. A. Panizzo, D. G. Gadian, J. C. Sowden, J. A. Wells, M. F. Lythgoe, and P. Ferretti, "Monitoring ferumoxide-labelled neural progenitor cells and lesion evolution by magnetic resonance imaging in a model of cell transplantation in cerebral ischaemia," F1000Res, vol. 2, p. 252, 2013.

[35] C. Heyn, C. V. Bowen, B. K. Rutt, and P. J. Foster, "Detection threshold of single spio-labeled cells with fiesta," Magn Reson Med, vol. 53, no. 2, pp. 312-20, 2005.

[36] P. Verdijk, T. W. Scheenen, W. J. Lesterhuis, G. Gambarota, A. A. Veltien, P. Walczak, N. M. Scharenborg, J. W. Bulte, C. J. Punt, A. Heerschap, C. G. Figdor, and I. J. de Vries, "Sensitivity of magnetic resonance imaging of dendritic cells for in vivo tracking of cellular cancer vaccines," Int J Cancer, vol. 120, no. 5, pp. 978-84, 2007.

[37] E. M. Shapiro, S. Skrtic, K. Sharer, J. M. Hill, C. E. Dunbar, and A. P. Koretsky, "Mri detection of single particles for cellular imaging," Proc Natl Acad Sci U S A, vol. 101, no. 30, pp. 10901-6, 2004.

[38] C. Heyn, J. A. Ronald, L. T. Mackenzie, I. C. MacDonald, A. F. Chambers, B. K. Rutt, and P. J. Foster, "In vivo magnetic resonance imaging of single cells in mouse brain with optical validation," Magn Reson Med, vol. 55, no. 1, pp. 23-9, 2006.

\section{AppendixA. Deriving Uncertainty of the Improved CISSCO Method}

Error propagation is used to determine the uncertainty of the modified CISSCO method presented in this work. The equation used to solve for the magnetic moment is given by Eq. 4. The variation of Eq. 4 leads to

$$
\frac{\delta p}{|p|}=\frac{1}{|D|} \sqrt{\left(\delta\left(\left|S_{2}-S_{3}\right|^{2}\right)\right)^{2} \frac{\left|f_{12}\right|^{4}}{p^{2}}+\left(\delta\left(\left|S_{1}-S_{2}\right|^{2}\right)\right)^{2} \frac{\left|f_{23}\right|^{4}}{p^{2}}}
$$

where

$$
D=\frac{\partial\left|f_{12}\right|^{2}}{\partial p}\left|S_{2}-S_{3}\right|^{2}-\frac{\partial\left|f_{23}\right|^{2}}{\partial p}\left|S_{1}-S_{2}\right|^{2}
$$

Note that $f_{i j}$ is given in Eq. 2 and the uncertainty of $\left|S_{i}-S_{j}\right|^{2}$ is defined as

$$
\delta\left(\left|S_{i}-S_{j}\right|^{2}\right)=\sqrt{\left(\eta_{i j}\left|S_{i}-S_{j}\right|^{2}\right)^{2}+\frac{16 \pi}{3} \sigma^{2}\left(R_{i}^{3}-R_{j}^{3}\right) \Delta x \Delta y \Delta z\left|S_{i}-S_{j}\right|^{2}}
$$

where $\eta_{i j}$ represents the systematic error of $\left|S_{i}-S_{j}\right|^{2}$ and $\sigma^{2}$ is the variance of the MR signal. We have assumed that the real part and imaginary part of the MR image has the same variance, but their Gaussian noise is independent. Equation A.3 is derived from the error propagation method. 
The systematic error $\eta_{i j}$ is equal to the difference between the summed $\left|S_{i}-S_{j}\right|^{2}$ from images and the theoretical $\left|S_{i}-S_{j}\right|^{2}$ calculated from the integrals given in [30], divided by the theoretical $\left|S_{i}-S_{j}\right|^{2}$. The partial derivatives of $\left|f_{i j}\right|^{2}$ with respect to $p$ are

$$
\frac{\partial\left|f_{i j}(p)\right|^{2}}{\partial p}=2 \operatorname{Re}\left(f_{i j}(p)\right) \frac{\partial \operatorname{Re}\left(f_{i j}(p)\right)}{\partial p}+2 \operatorname{Im}\left(f_{i j}(p)\right) \frac{\partial \operatorname{Im}\left(f_{i j}(p)\right)}{\partial p}
$$

The derivative of the real part of $f_{i j}(p)$ is given by Eq. 14 in [30] and the derivative of its imaginary part is

$$
\frac{\partial \operatorname{Im}\left(f_{i j}(p)\right)}{\partial p}=2 \int_{1}^{\frac{R_{i}^{3}}{R_{j}^{3}}} \frac{d x}{x}\left[\cos \left(x \frac{p}{R_{i}^{3}}\right)-\cos \left(2 x \frac{p}{R_{i}^{3}}\right)\right]+\int_{-1}^{2} \frac{d x}{x}[2-(2-x) \sqrt{1+x}]\left[\cos \left(x \frac{p}{R_{j}^{3}}\right)-\cos \left(x \frac{p}{R_{i}^{3}}\right)\right]
$$

